\title{
Sur la Structure des Amours (1552) de Ronsard
}

\author{
ROBERT MÉLANÇON
}

Dans un article qui marque une date pour la lecture des recueils de sonnets de la Renaissance française, Michel Dassonville a étudié la structure des Amours en suivant les diverses transformations que Ronsard a fait subir à son texte dans les éditions successives qu'il en a données: additions, suppressions et permutations de sonnets ont permis de saisir les métamorphoses du projet de Ronsard et, à travers elles, les différentes structures auxquelles il s'est arrêté tour à tour. ${ }^{1}$ Mais la méthode adoptée par Michel Dassonville pour établir sa démonstration ne lui a pas permis de décrire les Amours de 1552, l'état premier du texte et le point de départ de cet immense travail de recomposition: le texte originel ne peut être, par définition, comparé à un état antérieur qu'il transformerait. C'est la structure de ce recueil de 1552 que je me propose de décrire ici, en excluant toute considération des modifications que Ronsard lui a fait subir par la suite afin de le lire, autrement que comme la préfiguration des versions ultérieures, comme une oeuvre achevée, qui possède sa propre cohérence.

$$
* * *
$$

Le texte des Amours comprend, en 1552, un sonnet liminaire intitulé Voeu, une suite de 182 sonnets, une chanson (Las, je n'eusse jamais pensé) et une "amourette" (Petite Nymphe folastre). Si on tient compte du fait que la chanson et l'amourette forment un appendice ajouté après la mention "Fin des sonetz," on peut considérer qu'il s'agit d'un recueil entièrement composé de sonnets comme L'Olive, dont Du Bellay avait publié la deuxième édition augmentée en 1550. Puisque Ronsard va, par la suite, toujours intégrer à ses sonnets d'autres types de poèmes, élégies et chansons notamment, les Amours de 1552 constituent son seul recueil homogène. Paradoxalement c'est celui où l'influence des thèmes et du style pétrarquistes se fait peut-être le plus sentir qui s'écarte le plus nettement sur ce point du modèle pétrarquien du 
Canzoniere, où la suite des sonnets se trouve coupée de place en place par des chansons, des sextines, des ballades et des madrigaux. Tous ces sonnets sont écrits en décasyllabes et, par la disposition de leurs rimes et l'alternance des féminines et des masculines, ils se laissent réduire à quatre types formels:
$A^{m} B^{f} B^{f} A^{m}$
$A^{m} B^{f} B^{f} A^{m}$
$C^{f} C^{f} D^{m}$
$E^{f} E^{f} D^{m}$
$A^{m} B^{f} B^{f} A^{m}$
$A^{m} B^{f} B^{f} A^{m}$
$C^{f} C^{f} D^{m}$
$E^{f} D^{m} E^{f}$
$A^{f} B^{m} B^{m} A^{f}$
$A^{f} B^{m} B^{m} A^{f}$
$C^{m} C^{m} D^{f}$
$E^{m} E^{m} D^{f}$
$A^{f} B^{m} B^{m} A^{f}$
$A^{f} B^{m} B^{m} A^{f}$
$C^{m} C^{m} D^{f}$
$E^{m} D^{f} E^{m}$

Huit seulement, qui ne respectent pas l'alternance des rimes féminines et masculines entre le deuxième quatrain et le premier tercet, échappent à ce cadre. On sait que Ronsard s'est astreint à cette régularité pour faciliter leur mise en musique: tous les sonnets du même type formel peuvent être chantés sur le même air, et le supplément musical publié en appendice aux Amours en 1552 donne la liste de tous ceux qui peuvent l'être sur l'une ou l'autre des mélodies qu'il comprend. ${ }^{2}$

Mais il en résulte une autre conséquence que la facilité à les chanter, sur laquelle on n'a pas suffisamment attiré l'attention: une très grande unité formelle caractérise les Amours de 1552 dont les 182 sonnets, tous en décasyllabes, se répartissent en deux classes si on considère la disposition de leurs rimes dans les tercets où alternent les schémas mis au point par Marot et Peletier, et en quatre classes si on tient compte aussi de la répartition des féminines et des masculines. Les mêmes critères appliqués aux 115 sonnets de L'Olive déterminent plus de soixante types différents. Le recueil de Ronsard, au lieu d'une collection de pièces disparates, forme un édifice prosodique de proportions monumentales. Cette régularité métrique signale une recherche qui gouverne toute la composition. L'unité formelle n'est que la manifestation la plus immédiate, à la surface du texte, de l'organisation des thèmes, des figures et des styles qui confère à ce canzoniere sa cohérence.

Les thèmes développés dans ces quelques 2500 vers restent relativement peu nombreux même si leur expression est variée: la rencontre de la dame ${ }^{3}$ (II, LIV), la description de ses beautés (XVIII, CV), de ses yeux (XXV, LXIII), de ses cheveux (LXXVI, LXXVIII), de ses seins (CLX), la naissance de l'amour de Ronsard ${ }^{4}$ (III), l'expression des désirs que cet amour suscite (XX, XCVII) et de la souffrance qu'entraîne leur insatisfaction (XI, XLVII), la recherche de compensations imaginaires ou oniriques (XXIX, CLIX), la nostalgie de la liberté perdue sous le joug d'Amour (CLXIV, CLXXX), la sublimation du désir dans une ascèse tournée vers la perfection spirituelle (CXXXIX, CLXXIV). Bien que ces 
sonnets parlent toujours de la même passion absolue et qu'ils en évoquent de place en place quelques épisodes - par exemple la dame aperçue au milieu d'un pré fleuri occupée à confectionner un bouquet (CXXXI), une blessure de Ronsard qu'elle soigne (CXXXVII), une maladie qui la retient au lit (CLXI), le don d'une mèche de cheveux (CLII, CLXXVIII) - ils ne développent pourtant pas un récit.

Le relevé complet des mentions de temps et des dates reste étonnamment bref:

L'an est passé, le vingtuniesme jour

Du mois d'Avril, que je vins au sejour

De la prison, où les Amours me pleurent....

(XIV, v. 9-11)

Soubz le cristal d'une argenteuse rive,

Au mois d'Avril, une perle je vy....

$$
\text { (LXX, v. 1-2) }
$$

Bien que six ans soyent jà coulez derriere,

Depuis le jour que l'homicide trait

$\mathrm{Au}$ fond du coeur m'engrava le portrait

D'une humblefiere, \& fierehumble guerriere. ...

(LXXXVIII, v. 1-4)

L'an mil cinq cent contant quarante \& six,

Dans ses cheveux une beauté cruëlle,

...

Lia mon cuoeur de ses graces épris.

Lors je pensoy...

...

Que les crespons de leur blonde cautelle

Deux ou trois jours sans plus me tiendroyent pris:

L'an est passé, \& l'autre commence ores

Où je me voy plus que devant encores

Pris dans leurs retz. ...

(XCVIII, v. 1-2, 4-5, 7-11)

Depuis cinq ans dedans ce beau verger,

Je voys balant avecque faulx danger

Soubz la chanson d'Allegez moy Madame....

(CXXXVI, v. 9-11)

J'alloy roulant ces larmes de mes yeulx,

Or plein de doubte, ores plein d'esperance

Lors que Henry, loing des bornes de France,

Vangeoyt l'honneur de ses premiers ayeulx,

Lors qu'il trenchoyt d'un bras victorieux

Au bord du Rhin l'Espaignolle vaillance... .

(CLXXXII, v. 1-6) 
C'est trop peu pour fournir le cadre chronologique d'une narration suivie, d'autant que ces dates se succèdent en désordre (Bien que six ans soyent jà coulez. .., L'an est passé. .., Depuis cinq ans. . . ) et que leur exactitude reste suspecte, Ainsi, Ronsard aurait rencontré Cassandre un 21 avril, comme il aurait rencontré, plus tard, Marie un 20 avril; de même Pétrarque avait rencontré Laure un 6 avril, ${ }^{5}$ et Baiff sa Francine "le mois devant Avril." 6 De toute évidence, ce mois d'avril n'est pas une date mais un trope associant la naissance de l'amour au renouveau printanier dans des métaphores florales et lumineuses. ${ }^{7}$ Si des indications de ce genre étaient suffisamment abondantes, le recueil pourrait développer une sorte de récit archétypal ou simplement conventionnel, dont les divers épisodes seraient fixés par le code d'une tradition, mais elles restent trop fragmentaires et dispersées pour former un réseau cohérent. L'innamoramento au mois d'avril, repris par l'intermédiaire de Pétrarque des reverdies provençales, marque l'ouverture d'un espace de métaphores plutôt que le commencement d'une histoire. Les autres indications de temps ne suggèrent pas plus l'ébauche d'un récit. Dans le sonnet CXXXVI, les "cinq ans" du neuvième vers dérivent de la "danse des cinq pas" dans laquelle l'amoureux, après avoir été séduit par "Belacueil," se trouve entraîné par "faulx danger":
Hà, Belacueil, que ta doulce parolle
Vint traistrement ma jeunesse offenser
Quand au premier tu l'amenas dancer,
Dans le verger, l'amoureuse carolle.
Depuis cinq ans dedans ce beau verger,
Je voys balant avecque faulx danger,
Soubz la chanson d'Allegez moy Madame:
Le tabourin se nommoit fol plaisir,
La fluste erreur, le rebec vain desir,
Et les cinq pas la perte de mon ame.

(CXXXVI, v. 1-4. 9-14)

Ces cinq années allégoriques, dans un contexte saturé de références à ce monument d'allégories qu'est le Roman de la Rose, n'ont pas tant pour fonction d'indiquer qu'un espace de temps délimité s'est écoulé que de marquer une durée longue. L' "amoureuse carole" de cinq ans transforme de façon hyperbolique la danse des cinq pas. Au lieu d'une date, il s'agit, là encore, d'un trope. ${ }^{8}$ Quant aux "six ans jà coulez" du sonnet LXXXVIII, on les retrouve dans l' "Elégie à Marie" du Second livre des Amours de 1560:

Or les Dieus en feront cela qu'il leur plaira,

Si est-ce que ce livre après mille ans dira

Aux hommes, \& aus tems, \& à la renommée

Que je vous ay six ans plus que mon coeur aimée. ${ }^{9}$ 
Et, presque mot pour mot, dans l' "Elégie à Hélène" ajoutée aux Sonetz pour Helene en 1584:

Six ans estoient coulez, \& la septiesme annee

Estoit presques entiere en ses pas retournee.... ${ }^{10}$

Il serait pour le moins étonnant que la durée de trois passions successives ait toujours été la même, et ces six années sont probablement elles aussi la figure d'une longue durée plutôt qu'une indication temporelle précise. ${ }^{11}$

Restent les sonnets XCVIII et CLXXXII, qui marquent les limites de l'espace temporel à l'intérieur duquel se situent les Amours: de 1546 à 1552. C'est bien d'un espace qu'il s'agit. Entre ces dates, le temps, suspendu, cesse de couler et s'étale dans le texte sous la forme d'un présent perpétuel. La plupart des sonnets sont écrits au présent de l'indicatif parce que ce qui y est raconté est toujours en train de s'accomplir. Le passage des jours et des années n'est pas marqué parce qu'il ne peut pas modifier l'état amoureux. Dans les Amours, la passion n'a pas d'histoire. Elle ne progresse pas par un enchaînement de phases successives, où elle naîtrait, croîtrait, entraînerait la souffrance du désir insatisfait et sans cesse plus pressant, puis sa satisfaction ou sa sublimation ou son épuisement. Tout y est donné simultanément, plaisir et souffance, bonheur d'être captif d'Amour et nostalgie de la liberté, désir sensuel et sublimation néoplatonicienne:

J'espere \& crains, je me tais \& supplie,

Or je suis glace, $\&$ ores un feu chault,

J'admire tout, \& de rien ne me chault,

Je me delace, \& puis je me relie.

Rien ne me plaist si non ce qui m'ennuye,

Je suis vaillant, \& le cuoeur me default,

J'ay l'espoir bas, j'ay le courage hault,

Je doubte Amour, \& si je le deffie.

Plus je me picque, \& plus je suis restif,

J'ayme estre libre, \& veulx estre captif,

Cent foys je meur, cent foys je prens naissance.

Un Promethée en passions je suis,

Et pour aymer perdant toute puissance,

$\mathrm{Ne}$ pouvant rien je fay ce que je puis.

(XII)

Chaque sonnet reste clos sur lui-même. Il forme un tout autonome, évoquant une scène ou un moment, presque un état, dans une durée coupée de tout enchaînement narratif. Les accidents de cette passion ne forment pas les épisodes d'une histoire en devenir: événements purs, ils restent contenus dans le cadre étroit des sonnets qui se succèdent les uns aux autres dans une démarche tout à fait ponctuelle. L'espace de 
quatorze vers, quelque chose a lieu et s'offre à la lecture comme un fait complet, qui se suffit à lui-même, indépendant de ceux qui le précèdent ou le suivent, sans être déterminé par eux et sans les déterminer. Ainsi, après le parallèle entre Amour et Mars développé au sonnet CXLVIII, le CXLIX $^{\mathrm{e}}$ évoque le plaisir intense qu'a ressenti l'amoureux, gratifié par sa dame d'un "petit ris de l'oeil" et qui tente d'imaginer, par raisonnement a fortiori, "quel paradis. . apporteront les nuictz. . .dans le sein de Madame"; puis le CL $\mathrm{C}^{\mathrm{e}}$ raconte au passé, dans un décor naturel d'un symbolisme appuyé, l'enlèvement de la dame par un Centaure qui la ravit à l'amoureux au moment où il allait lui déclarer ses sentiments. Aucune continuité ne s'impose entre ces sonnets. Ils se succèdent en s'abolissant tour à tour comme des points qu'aucun trait ne relie. Il s'agit là d'un exemple extrême. Le plus souvent, en effet, on peut déceler un rapport quelconque, plus ou moins évident, entre deux sonnets qui se suivent. Le CLI ${ }^{\mathrm{e}}$ décrit une maison "veufve. . des beaulx yeulx de Madame" et dont les riches ornements ne peuvent toucher l'amoureux parce qu'il souffre de continuer à y "voyr. . .ceste Dame, \& l'ouyr,.. . tousjours. . . dans sa pensée"; développant ainsi le thème de l'absence de la maîtresse, il s'enchaîne avec une certaine logique au récit de son enlèvement par un Centaure au $\mathrm{CL}^{\mathrm{e}}$. Mais la relation entre ces deux sonnets ne se trouve indiquée nulle part dans leur texte, et c'est au lecteur qu'il appartient de les rattacher l'un à l'autre dans la pause qui sépare le quatorzième vers de l'un du premier vers de l'autre. Un silence vient scander la lecture entre chaque poème, et le recueil apparaît comme une succession de textes clos sur eux-mêmes, chacun formant un bloc compact de significations encadré par quatre marges qui l'isolent sur la page.

La forme même du sonnet, serrée, hypercodifiée, saturée de relations internes denses, impose cette fragmentation. Comme l'a écrit Valéry, "le sonnet est fait pour le simultané; quatorze vers simultanés, et fortement désignés comme tels par l'enchaînement et la conservation des rimes: type et structure de poème stationnaire." ${ }^{2}$ Un recueil de sonnets se définit donc comme le rassemblement de textes que la logique de leur forme constitue en entités closes, paradoxe qu'a bien décrit Jean Rousset: "intégré dans le canzoniere, le sonnet accepte de perdre sa nature de monade pour se fondre dans un tout." ${ }^{13}$ Entre le réseau de relations internes que détermine sa forme même et l'ensemble de relations externes qu'entraîne son insertion dans un recueil, le sonnet apparaît comme un lieu de tensions et comme le croisement de plusieurs lignes de force contradictoires. De là sans doute la puissance esthétique et l'extraordinaire fortune du genre "recueil de sonnets": l'unité doit y être gagnée sur des forces de dispersion très grandes et doit s'affirmer d'autant plus nettement qu'elle doit les surmonter sans pouvoir les abolir tout à fait. Si unitaire que soit son propos, en effet, un recueil de sonnets implique toujours une certaine 
fragmentation. Sans doute est-t-il utile de rappeler ici que Pétrarque, dès le premier vers du Canzoniere, désigne ses poèmes par l'expression "rime sparse." 14

On n'en conclura pas nécessairement qu'il ne peut y avoir de recueil de sonnets vraiment structuré, organisé, centré autour d'un foyer. Mais cette structure sera toujours le lieu de contradictions et de discontinuités; elle devra être gagnée sur la dispersion en fragments autonomes, bons à être détachés dans les anthologies (ce n'est pas un hasard si celles-ci contiennent une proportion si élevée de sonnets). Il est même possible qu'un recueil développe un récit linéaire, comme l'a montré Jean Rousset dans son analyse de L'Olive. ${ }^{15}$ Mais ce récit sera toujours brouillé, rompu, discontinu à cause de son découpage en sonnets: Rousset n'a pu mettre en lumière l'itinéraire tracé par Du Bellay qu'en rapprochant, de façon à les faire contraster, l'ouverture et le finale de L'Olive.

On a vu qu'il n'y a pas de récit dans les Amours, ni la progression d'un itinéraire même confus et discontinu. Le sonnet l'ouverture annonce une description du tourment et du bonheur d'aimer:

\footnotetext{
Qui voudra voyr comme un Dieu me surmonte,

Comme il m'assault, comme il se fait vainqueur,

Comme il r'enflamme, \& r'englace mon cuoeur,

Comme il reçoit un honneur de ma honte,

Qui voudra voir une jeunesse prompte

A suyvre en vain l'object de son malheur,

Me vienne voir: il voirra ma douleur,

Et la rigueur de l'Archer qui me donte.

Il cognoistra combien la raison peult

Contre son arc, quand une foys il veult

Que nostre cuoeur son esclave demeure:

Et si voirra que je suis trop heureux,

D'avoir au flanc l'aiguillon amoureux,

Plein du venin dont il fault que je meure.
}

Et l'avant-dernier sonnet (le dernier sert à dater le recueil et se trouve de ce fait un peu en marge) reprend les mêmes données, en repoussant la possibilité même d'une conclusion:

\footnotetext{
Veu la douleur qui doulcement me lime,

Et qui me suit compaigne, paz à paz,

Je cognoy bien qu'encor' je ne suis pas,

Pour trop aymer, à la fin de ma ryme.

Dame, l'ardeur qui de chanter m'anime,

Et qui me rend en ce labeur moins las,

C'est que je voy qu'aggreable tu l'as,

Et que je tien de tes pensers la cyme.
}

Je suis vrayment heureux \& plusque heureux 
De vivre aymé \& de vivre amoureux

De la beaulté d'une Dame si belle:

Qui list mes vers, qui en fait jugement,

Et qui me donne à toute heure argument

De souspirer heureusement pour elle.

(CLXXXI)

On en déduirait hâtivement toutefois que le recueil de Ronsard n'est pas organisé. Une indéniable unité de ton, perceptible à une lecture même distraite, découle de la mise en oeuvre incessante d'un ensemble très restreint de motifs, de thèmes, de registres stylistiques, qu'on peut définir rapidement comme une interprétation du pétrarquisme. ${ }^{16}$ Entre le premier sonnet et le dernier, la reprise constante des mêmes éléments tisse entre les divers poèmes des rapports multiples et très variés qui composent une structure d'une grande fermeté. C'est cette structure qui donne au recueil de Ronsard sa signification et qui en fait autre chose que la répétition pure et simple de lieux communs.

La première série de rapports qu'entretiennent entre eux les sonnets des Amours, et la plus évidente, est déterminée par l'ordre selon lequel ils se succèdent; même s'il ne dessine pas la courbe d'un récit qui embrasserait tout le recueil, et même s'il n'est pas significatif en ses moindres détails, il n'est pas indifférent. Une lecture suivie permet de déceler des séquences plus ou moins longues de sonnets liés les uns aux autres et dont l'enchaînement développe un thème qu'aucun, isolément, ne saurait entièrement contenir. Les sonnets XXIX et XXX, par exemple, amplifient le thème du songe érotique ébauché dans les derniers vers du sonnet XXVIII. Autre exemple, les sonnets CLXVIII-CLXX se présentent comme des variations sur les larmes de la dame dont il aurait été impossible de condenser la richesse ingénieuse en quatorze vers.

D'autres séquences sont plus complexes. Ainsi les sonnets II à VI développent parallèlement, en sorte qu'ils se mettent mutuellement en relief, le thème de la beauté superlative de la dame et celui de la naissance de l'amour chez le poète: après un portrait général de Cassandre, qui n'évoque aucune qualité particulière mais accumule les hyperboles les plus vagues ("des beautez les plus belles") et un récit tout aussi général de la séduction de l'amoureux (II), le sonnet III propose un récit plus élaboré de la première rencontre et de l'amour qui a foudroyé le poète; le $\mathrm{IV}^{\mathrm{e}}$, à travers un luxe d'allusions érudites à la guerre de Troie, tente de définir la nature de cet amour; enfin, le $\mathrm{V}^{\mathrm{e}}$ et le $\mathrm{VI}^{\mathrm{e}}$ reprennent le portrait hyperbolique de la dame et l'analyse de la naissance de la passion sur des modes différents de façon à épuiser provisoirement ces thèmes.

Dernier exemple, la série des sonnets LXXX à C développe de façon extrêmement sinueuse le thème des souffrances de l'amoureux, en y 
mêlant d'autres thèmes secondaires qui le colorent diversement, et en variant les points de vue selon lesquels il est successivement présenté. Le sonnet LXXX inaugure cette séquence en reprenant le récit de la naissance de l'amour sous la forme du vol du coeur du poète, qui est allé se "cacher dans [les] ondes d'ivoire, Et soubz l'abri [des] flancz amoureux" de la dame, laissant son légitime possesseur "seul, palle \& froid... Comme un esprit qui fuit de son tombeau." Suivent six sonnets sur le mal d'amour considéré tantôt négativement comme une agonie où tout bien se transforme en mal (LXXXI, LXXXII, LXXXV, LXXXVI), tantôt positivement comme un bonheur paradoxal qui s'alimente de souffrance (LXXXIII, LXXXIV). Le sonnet LXXXVII interrompt ces lamentations par un splendide portrait de la beauté printanière de la dame, repris au sonnet LXXXVIII où il confère une nouvelle intensité au paradoxe du bonheur qu'entraîne le malheur d'aimer sans retour: malgré les mille morts qu'il endure, l'amoureux est "heureux d'avoir veu la lumière... De son beau front," parce que cette contemplation de la beauté porte en elle-même sa propre récompense. ${ }^{17} \mathrm{Par}$ contraste, le sonnet LXXXIX, l'un des plus sombres du recueil, montre l'amoureux "franc de raison, esclave de fureur," en victime de sentiments qui se tournent contre lui et qui, par comparaison implicite au mythe d'Actéon, "comme mastins affamez de repaistre. . se paissent de leur maistre"; le sonnet suivant tempère cette violence en établissant un parallèle entre Ronsard, qui tente de guérir son mal par la poésie, et Apollon, "ce grand Dieu de la lyre," lui-même amoureux malheureux de Cassandre, la princesse troyenne. Le sonnet XCI énumère une série d'objets liés à la dame, dont l'évocation baigne l'amoureux "de deux ruisseaux de larmes"; le XCII oppose à sa souffrance le bonheur de Roger qui, dans le Roland Furieux de l'Arioste, obtient immédiatement les faveurs d'Alcine, et le XCIII ${ }^{\mathrm{e}}$ le met en scène fuyant la société dans une nature sauvage où il peut s'abandonner sans contrainte à sa douleur. Les sonnets XCIV et XCV interrompent à nouveau la description de ses malheurs par deux évocations des beautés de cette dame si rigoureuse: le premier, fondé sur des métaphores empruntées à la nature, prolonge le sonnet XCIII, et le second, qui se termine par une allusion aux "beaulx yeulx" qui l'ont tué, ramène au thème dominant de la séquence. Après l'appel à un ami pris à témoin de l'étendue de son malheur au sonnet XCVI, l'amoureux exaspéré demande à la dame, au sonnet XCVII, de céder à ses avances ou de se refuser une fois pour toutes "sans tant...desguiser." Le $\mathrm{XCVIII}^{\mathrm{e}}$ marque un retour en arrière au commencement de cette passion qui le nourrit d'une "esperance vaine," le XCIX ${ }^{\mathrm{e}}$ renouvelle à la dame l'offrande de son coeur, et le $\mathrm{C}^{\mathrm{e}}$ exprime l'espoir de voir un jour son désir satisfait, après quoi il mourrait aussitôt "yvre" d'un bonheur si excessif. 
Les limites de ces séquences ne sont pas tranchées, et elles ne peuvent pas l'être de façon absolument nette. Elles ne constituent pas des chapitres caractérisés, logiquement articulés les uns aux autres, mais des zones plus ou moins bien délimitées, où on peut observer avec une intensité plus grande qu'ailleurs tel thème ou tel registre de métaphores. A l'intérieur d'une séquence un peu longue, certains sonnets resteront en marge du mouvement d'ensemble même s'ils entretiennent avec leurs voisins immédiats des relations fondées sur des thèmes secondaires, sur la possession commune d'une métaphore ou, même, d'un mot particulièrement important. Ainsi les sonnets XLIV et XLV, qui diffèrent par presque tout ont néanmoins en commun l'image de la navigation qui établit entre eux une correspondance plastique; pour les sonnets $\mathrm{L}$ et LI, c'est le mot "pré" qui fait office de lien, mot surdéterminé dans ce recueil puisque Cassandre Salviati à laquelle il s'adresse était devenue Mademoiselle de Pré par alliance avec Jehan Peigné, seigneur de Pré. Ailleurs un sonnet aura pour fonction de clarifier quelque aspect demeuré obscur dans celui qui le précède, comme le $\mathrm{CXXV}^{\mathrm{e}}$ qui précise le sens qu'il convient d'accorder à l'étonnante utilisation du mythe de Narcisse dans le dixième vers du $\operatorname{CXXIV}^{\mathrm{e}}$. Ou encore, il développera un thème ébauché dans celui qui le précède: un seul vers du XII ${ }^{\mathrm{e}}$ sonnet ("Un Prométhée en passions je suis") engendre au sonnet XIII tout un parallèle entre les souffrances de l'amoureux et Prométhée enchaîné au Caucase pour avoir ravi le feu du ciel, entre sa délivrance par Hercule et les faveurs de la dame qui mettront fin aux tourments de l'amour.

Cette organisation linéaire en séquences plus ou moins étendues n'est cependant pas le seul principe qui guide la composition, ni le plus important. Les sonnets s'inscrivent dans un réseau de relations qui n'est pas déterminé par la proximité ou l'ordre de succession. Chaque sonnet répond à plusieurs autres, qui sont souvent situés à une grande distance, auxquels il fait écho ou qu'il anticipe, dont il reprend les thèmes, les métaphores, les formes, pour les préciser, les réorienter ou les inscrire dans un autre contexte où ils vont prendre un autre sens.

La série des sonnets qui tracent le portrait de la dame, par exemple, dispersée sur toute l'étendue du recueil, scande la lecture et déploie un espace d'analogies qui se développe selon sa propre logique. Dès l'ouverture, aux sonnets II, III, V, VI, on voit apparaître les premiers éléments de ce portrait; on n'en trouve toutefois pas avant le $\mathrm{XVIII}^{\mathrm{e}}$ un exemple étendu aux quatorze vers du sonnet:

Un chaste feu qui les cuoeurs illumine,

Un or frisé de meint crespe annelet,

Un front de rose, un teint damoiselet,

Un ris qui l'ame aux astres achemine:

Une vertu de telles beaultez digne, 
Un col de neige, une gorge de laict,

Un cuoeur jà meur dans un sein verdelet,

En dame humaine une beaulté divine:

Un oeil puissant de faire jours les nuictz,

Une main forte à piller les ennuiz,

Qui tient ma vie en ses doitz enfermée,

Avecque un chant offensé doulcement

Ore d'un ris, or d'un gemissement:

De telz sorciers ma raison fut charmée.

(XVIII)

Pour l'essentiel, ces portraits reprennent la forme canonique du blason: l'énumération de traits ponctuels, simplement juxtaposés, unifiés par la répétition d'un même déterminant - ici l'article indéfini, ailleurs le vocatif "ô" (XLVI), le démonstratif (CX), le possessif (CLVI), voire l'absence de tout déterminant $(\mathrm{CV})$ - et rassemblés à la fin par un vers où ils trouvent une fonction syntaxique. La simplicité de cette forme assure son identification immédiate à chacune de ses occurrences: aux sonnets XXIII, LXXV, XCV, le lecteur retrouve un même ry thme, un même registre de métaphores, et chaque fois la lecture se suspend. Par delà les correspondances immédiates qu'il entretient avec les sonnets qui l'entourent, chaque portrait renvoie aux autres, en sorte que le recueil se replie sur lui-même. Comme toujours, la récurrence impose un rythme. La ligne des 182 sonnets ne se déroule donc pas de façon uniforme; elle s'anime de place en place, se reprend, recommence sans toutefois se répéter puisque chaque nouveau portrait assume et transforme tous ceux qui l'ont précédé.

La forme de base du blason fait l'objet d'un certain nombre de variations qui, dispersées elles aussi dans toute l'étendue du recueil, la mettent en communication avec d'autres réseaux formels ou sémantiques. Dans la tradition du portrait synthétique, Ronsard va décrire la dame comme la réunion des qualités les plus éminentes du panthéon: ${ }^{18}$

Quand au premier la Dame que j'adore

Vint embellir le séjour de noz cieulx,

Le filz de Rhée appella tous les Dieux,

Pour faire encor d'elle une aultre Pandore.

Lors A pollin richement la decore,

Or, de ses raiz luy façonnant les yeulx,

Or, luy donnant son chant melodieux,

Or, son oracle \& ses beaulx vers encore.

Mars luy donna sa fiere cruaulté,

Venus son ris, Dione sa beaulté,

Peithon sa voix, Ceres son abondance.

L'Aube ses doigtz \& ses crins deliez,

Amour son arc, Tethis donna ses piedz,

Cleion sa gloyre, \& Pallas sa prudence.

(XXXII) 
Cette fiction, dont on trouve une autre version au sonnet CVI, justifie la structure énumérative des portraits en présentant la beauté de la dame comme l'accumulation de beautés distinctes plus ou moins autonomes. D'autre part, en utilisant le mythe de Pandore, qu'il reprendra au sonnet LXXXV pour dénoncer la "malencontreuse \& meschante esperance" qui l'abuse, Ronsard établit l'origine divine de Cassandre. La passion qu'il éprouve pour elle prend les proportions d'un destin: elle doit fatalement engendrer ruine et malheur comme les maux échappés du coffre de Pandore mirent fin à l'âge d'or. La beauté est donc extrêmement ambiguë: sa seule contemplation porte en elle-même une récompense suffisante, elle libère l'âme et l'engage sur la voie de la perfection spirituelle en manifestant ici bas la présence du divin, mais elle sème aussi au coeur de son amoureux un souci qui ne cesse de le tourmenter et qui dissipe toute joie. Elle fascine l'amoureux qui ne peut détourner d'elle sa pensée et qui se trouve condamné à un ressassement infini. D'où ces sonnets qui présentent tel ou tel détail agrandi: le teint (LIV), les cheveux (LXXVI, LXXVIII), les yeux (XXV, LXXII, CXXII), les seins (CLX). D'où aussi, dans les contex tes les plus divers, ces reprises en quelques vers d'éléments du blason qui viennent orienter le développement d'autres thèmes: aux sonnets XVII et XCIII, de brèves énumérations unissent les souffrances de l'amoureux et sa fuite dans la solitude au pouvoir de la beauté de la dame, et le sonnet LV marque la signification de l'éloge de cette beauté dans la rédaction des poèmes. C'est la répétition incessante des mêmes figures dans l'ensemble du recueil qui fait sens. La contemplation émerveillée de la beauté fait place peu à peu à une obsession malheureuse: le texte recommence, revient sur lui-même, déploie un espace d'allusions et d'échos qui brise la succession des sonnets. Un autre ordre s'impose, ou plutôt l'ordre change de sens: parcourant la succession des sonnets, on n'a plus à suivre simplement une ligne qui relierait le premier au dernier, mais à démêler un écheveau de rapports qui se modifie à chaque moment. Il y a en effet plusieurs séries qui ne cessent de se croiser dans une véritable polyphonie. Par exemple, celles qui rassemblent des sonnets d'inspiration néoplatonicienne (XXVI, XLII, LVI, LXII, LXIX, CXXX, CXXXIX, CXLVI, CLXXIV) et des sonnets où s'exprime une sensualité exigeante (XX, XXX, LXVIII, XCVII, CII, CVIII, CXVII, CXXIX, CLIX) dialoguent tout au long du texte des Amours. Aucune ne domine vraiment. Le désir physique s'exprime avec vigueur et précision dans plusieurs sonnets. Le LXVII ${ }^{\mathrm{e}}$, en particulier, est remarquable en ce qu'il renverse la hiérarchie des sens établie par Ficin, selon qui "Les sens nobles (la vue et l'ouie) seuls capables de percevoir la beauté s'opposent aux sens matériels (le goût, le tact, l'odorat) qui ne peuvent être liés qu'à l'amour sensuel"; ${ }^{19}$ on croirait lire, dans les tercets, une glose polémique du Commentaire à Platon de l'humaniste florentin: 
Ny ce beau chef, ny ces yeulx, ny ce front,

Ny ce doulx ris, ny ceste main qui fond

Mon cuoeur en source, \& de pleurs me fait riche,

$\mathrm{Ne}$ me sçauroyent de leur beau contenter,

Sans esperer quelque foys de taster

Ton paradis, où mon plaisir se niche.

(LXVII, v. 9-14)

Mais il est remarquable que l'élan vers le ciel des idées ne s'exprime pas avec moins d'impétuosité:

Je veus brusler pour m'en voler aux cieux,

Tout l'imparfait de ceste escorce humaine,

M'eternisant, comme le filz d'Alcméne,

Qui tout en feu s'assit entre les Dieux.

Ja mon esprit chatouillé de son mieux,

Dedans ma chair, rebelle se promeine,

Et ja le bois de sa victime ameine

Pour s'enflammer aux rayons de tes yeulx.

O sainct brazier, ô feu chastement beau,

Las, brusle moy d'un si chaste flambeau

Qu'abandonant ma despouille cognue,

Nét, libre, \& nud, je vole d'un plein sault,

Oultre le ciel, pour adorer là hault

L'aultre beaulté dont la tienne est venue.

(CXXXIX)

Partagé entre le désir et l'élan ascétique, l'amoureux ne choisit pas; il passe de l'un à l'autre avec violence, sans jamais hésiter, accumulant les contradictions. L'opposition du platonisme et de la sensualité dans Les Amours est le conflit de deux pulsions également exigeantes. Si elles ont quelque chose en commun, c'est l'impatience avec laquelle elles se manifestent toutes deux, interdisant tout compromis aussi bien que tout choix. L'appel à la perfection spirituelle qui libérerait l'âme de la prison du corps n'assume pas le désir en le sublimant parce qu'il commence par le nier en vain, et le désir sensuel ne parvient pas non plus à abolir la nostalgie de cette "autre beaulté dont la tienne est venue." Ronsard n'a pas adopté une métaphysique comme Pontus de Tyard, dont le Solitaire premier commente l'arrière-plan philosophique des poèmes des Erreurs amoureuses; il semble plutôt avoir tiré de la lecture de Marsile Ficin et de Léon L'Hébreu les éléments d'une dramaturgie sans issue. Le croisement des séries opposées de sonnets platoniciens et de sonnets sensuels dans Les Amours, où elles sont distribuées sensiblement de la même façon, instaure une tension constante entre deux principes opposés qui marquent les limites entre lesquelles se déploient tous les autres thèmes. Elles définissent un champ de forces qui jouent du premier au dernier sonnet. Entre la violence du désir physique et l'effort de purification 


\section{2 / Renaissance and Reformation}

intérieure qui conduit par l'amour à la contemplation de la beauté divine, les contradictions ne sont pas moins marquées au début qu'à la fin. Dès le sonnet XXI, déchiré par le conflit entre les exigences de sa chair et sa soif de perfection spirituelle, l'amoureux craint "que [sa] raison ne verse." Et nulle solution n'apparaît à la fin du recueil où les mêmes termes sont toujours en présence: si le sonnet CLXXIV décrit l'envol "jusqu'aux cieux" vers la vérité dont "ici bas" ne propose que les apparences décevantes, les sonnets CLXXVII et CLXXVIII reprennent l'éloge de la beauté de la maîtresse en des termes qui ne laissent aucun doute sur la nature sensuelle du désir qui s'y exprime.

D'autres thèmes moins importants sont aussi repris de place en place, disposés dans toute l'étendue du recueil où ils se greffent aux grandes séries qui viennent d'être évoquées. Ainsi l'amoureux se réfugie plusieurs fois dans une nature solitaire où rien ne le distraira de la rumination de sa malheureuse passion (IX, XCIII, CXXXIII), ou encore il s'abandonne régulièrement à un songe où son désir se donne libre cours (XXIX, XXX, LXI, CI, CLIX). Tous ces thèmes se contaminent les uns les autres au point de devenir indissociables. Leurs réapparitions périodiques plus ou moins fréquentes et les variations dont ils font l'objet les enrichissent d'une épaisseur sémantique de plus en plus grande à mesure que progresse la lecture. Celle-ci ne se déroule pas selon le temps linéaire d'un récit, elle explore l'espace polyphonique d'un réseau d'associations. Chaque sonnet en évoque plusieurs autres, qui éveillent eux-mêmes, de proche en proche, d'autres réponses comme autant d'harmoniques. En ce sens, les 182 sonnets ne se succèdent pas, ils se donnent à lire simultanément selon une mémoire inscrite dans la lettre même de leur texte.

Outre ces reprises thématiques, on rencontre, plus rarement il est vrai, des reprises formelles qui ne sont pas moins significatives. Une, en particulier, propose une véritable clé du recueil. On retrouve en effet au sonnet LIII la structure rhétorique très voyante du sonnet d'ouverture: ${ }^{20}$

Qui vouldra voyr dedans une jeunesse,

La beaulté jointe avec la chasteté,

L'humble doulceur, la grave magesté,

Toutes vertus, \& toute gentillesse:

Qui vouldra voyr les yeulx d'une deesse,

Et de noz ans la seule nouveauté,

De ceste Dame oeillade la beaulté,

Que le vulgaire appelle ma maistresse.

Il apprendra comme Amour rid \& mord,

Comme il guarit, comme il donne la mort,

Puis il dira voyant chose si belle:

Heureux vrayment, heureux qui peult avoyr

Heureusement cest heur que de la voyr,

Et plus heureux qui meurt pour l'amour d'elle.

(LIII) 
Le ton inaugural de ce sonnet n'est pas moins marqué que celui du premier, d'autant qu'il est suivi lui aussi d'une évocation de la naissance de l'amour (LIV). Ce nouveau commencement change le sens du début du recueil; il ne décrit pas le commencement de l'amour du poète pour sa dame; il marque un moment parmi d'autres de cette passion qui a toujours éfé là, qui ne cesse pas de naître et qui ne saurait s'achever parce qu'elle est antérieure à toute origine, inscrite de tout temps dans les astres qui guident les destins, consubstantielle à l'être même de l'amoureux:

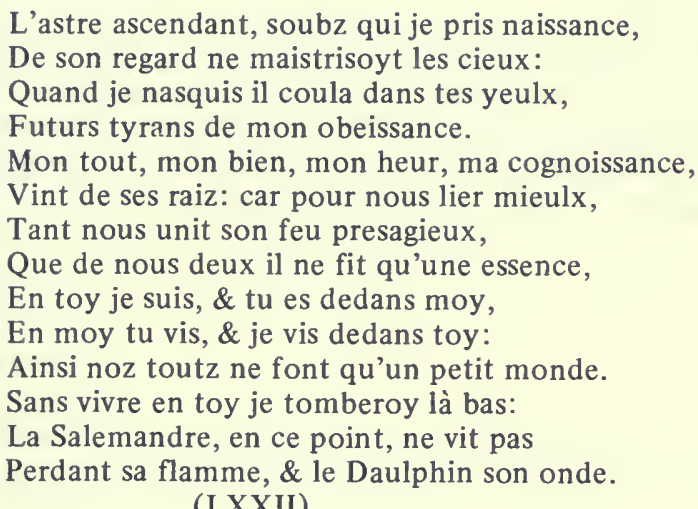
(LXXII)

Le livre doit pourtant commencer et s'achever, s'incarner en un nombre fini de pages où il enclorra cet amour qui n'a pas de bornes. Son début est donc un faux début, redoublé et annulé par ce redoublement après qu'on ait lu plus du quart des poèmes; et sa fin est une fausse fin qui repousse toute conclusion. Lorsque Ronsard écrit, dans l'avant-dernier sonnet de son livre: "Je cognoy bien qu'encor je ne suis pas/Pour trop aimer, à la fin de ma rime," il n'annonce pas tant une suite à son livre (l'édition augmentée des Amours en 1553, la Continuation et la Nouvelle Continuation en 1555-1556) qu'il ne marque le caractère infini de son entreprise. L'écriture est sans fin parce que l'amour est sans fin. Le poète-amoureux ne pourra pas plus toucher le "bout de sa rime" que la fin de son amour, et s'il manifestait la moindre velléité de "garir sa verve poetique," Amour saurait en le "martel[ant] de coups" lui faire entendre "que jamais on n'estrange. .l'arrest du ciel qui preside sur nous" (CLVII).

Sans début et sans fin véritables, le recueil se déploie donc, entre sa première et sa dernière pages, comme un réseau d'associations pratiquement infini. Les sonnets s'enchaînent en des séquences linéaires sans toutefois devenir les moments successifs d'un récit; ils entretiennent avec d'autres sonnets dont ils reprennent à distance les thèmes, les métaphores, les formes en les transformant et en les réorientant, des rapports multiples par lesquels le recueil se donne pres- 


\section{4 / Renaissance and Reformation}

que à lire dans sa totalité en chacun de ses points, Il est inépuisable parce que chaque sonnet qu'il contient est inépuisable par référence à l'ensemble. En évitant avec soin de disposer ses poèmes dans un ordre chronologique qui eût engendré une narration (un commencement, des épisodes, une fin) et en conférant à leur succession un rythme varié (sans doute faudrait-il parler de rythmes superposés) par l'entrecroisement de plusieurs systèmes d'échos, de reprises, de variations sur un nombre restreint de thèmes, Ronsard a réussi un livre vertigineux, qui ne cesse de se reprendre et de recommencer, où le sens se donne toujours en deçà ou au delà de la lettre du texte et de son environnement immédiat. Si jamais une oeuvre a pu être dite ouverte et fermement composée à la fois, c'est bien celle-là, structurée comme un espace d'associations toujours en mouvement, qui ne cesse de proposer de nouveaux parcours à la lecture. Aussi, cette lecture est-elle toujours inachevée.

\section{Université de Montréal}

Notes

1 Michel Dassonville, "Pour une interprétation nouvelle des Amours de Ronsard," BHR, XXVIII-2 (1966), pp. 241-70.

2 Cf. Brian Jeffery, "The Idea of Music in Ronsard's Poetry," in T.C. Cave (ed.), Ronsard the Poet (London: Methuen, 1973), pp. 209-39. Voir aussi les introductions aux éditions suivantes: Pierre de Ronsard, Les Amours, éd. H. et C. Weber (Paris: Garnier, 1963), pp. LXIV-LXVI, et P. de Ronsard, Oeuvres complètes, éd. P. Laumonier (Paris: Didier, "S.T.F.M.," 1957), tome IV, pp. xiii-xix. C'est le tex te de cette dernière édition qui sera cité au cours de cet article.

3 Le nom de Cassandre n'apparaît que trois fois dans les Amours en 1552: dans l'inscription grecque sous son portrait en tête du livre et dans les sonnets IV et XXXIII. Un autre nom de femme, Marguerite, y apparaît également trois fois: allusivement, au sonnet LXX, par un jeu de mots latinisant sur "perle," et, en toutes lettres, dans les sonnets LXXVII et CLXII. Le titre du recueil de 1552 (Les Amours/De P. de Ronsard/Vandomoys) est très général et le restera jusqu'en 1578, date à laquelle Ronsard "modifie[ra] complètement la signification de l'oeuvre en la centrant désormais sur le personnage de Cassandre" (M. Dassonville, op. cit., p. 265).

4 Il s'agit évidemment, comme partout dans les pages qui suivent, du sentiment qui est décrit dans les poèmes, non d'une avanture galante qui a pu avoir lieu ou non dans la réalité entre le Ronsard historique et Cassandre Salviati, la fille d'un banquier florentin. Pour une critique de la lecture biographique des recueils amoureux de Ronsard, cf. K.R.W. Jones, $P$. de Ronsard (New York: Twayne, "T.W.A.S." no. 132, 1970), pp. 42-44.

5 Rapprochements signalés dans P. de Ronsard, Oeuvres complètes, éd. Laumonier, Lebègue, Silver, tome XIX, Errata et Addenda (Paris: Didier, "S.T.F.M.,"1974), p. 45.

6 Baïf, Amours de Francine, Livre I, sonnet X (éd. Marty-Laveaux, t. I, p. 97).

7 Cf. sonnet CIII, v. 1-4:

Je parangonne à ta jeune beaulté,

Qui tousjours dure en son printemps nouvelle,

Ce moys d'Avril, qui ses fleurs renouvelle,

En sa plus gaye \& verte nouveaulté. 
8 Sur ce sonnet, cf. A. Adler, "The topos Quinque lineae sunt amoris used by Ronsard in Amours (1552) CXXXV1," BHR, XV-2 (1953), pp. 220-25.

9 "Elégie à Marie," v. 117-120, Ronsard, Les Amours, éd. H. et C. Weber, op. cit., p. 293.

10 “Elégie à Hélène," v. 1-2, idem, p. 483.

$11 \mathrm{Cf}$. aussi, à titre de confirmation, les Sonetz pour Helene, livre 11, sonnet VII, où Ronsard, en s'appuyant sur une loi hébraïque (Exode, XXI, 2), précise la valeur symbolique de sept années de "servage" amoureux.

12 Paul Valéry, Tel Quel, in Oeuvres, éd. J. Hytier (Paris: Gallimard, "La Pléiade," 1960), tome 11, p. 676.

13 Jean Rousset, L'Intérieur et l'Extérieur (Paris: José Corti, 1968), p. 13.

14 F. Petrarca, Il Canzoniere (Milano: Biet ti, 1966, p. 35), sonnet I, v. 1.

15 “...Une ample trajectoire qui suggère une théophanie: une divinité apparaît, séjourne sur terre, puis s'éloigne, désignant le ciel; entre deux, les déchirements, les épreuves de l'amant terrestre." - J. Rousset, op. cit., p. 14.

16 Cf. L. Keller, "Solo e pensoso, Seul et pensif, Solitaire et pensif, mélancolie pétrarquienne et mélancolie pétrarquiste," Studi Francesi, 49 (1973), 3-14.

17 Cf. G. Castor, "Petrarchism and the quest for beauty in the Amours of Cassandre and the Sonetz pour Helene," in T.C. Cave (éd.), Ronsard the Poet, op. cit., pp. 79-120.

18 Sur ce sonnet, cf. A.H. Schultz, "Ronsard's Amours XXXII and the Tradition of the Synthetic Lady," Romance Philology, 1 (1947), 125-35.

19 H. Weber, "Platonisme et sensualité dans la poésie amoureuse de La Pléiade," in Lumières de La Pléiade (Actes du $1 \mathrm{X}^{\mathrm{e}}$ Stage international d'Etudes Humanistes, Tours 1965), (Paris: Vrin, "De Pétrarque à Descartes," 1966), pp. 158-59.

20 Citć supra, p. 125. 